\title{
Inductance Model of Interdigitated Power and Ground Distribution Networks
}

\author{
Renatas Jakushokas, Student Member, IEEE, and Eby G. Friedman, Fellow, IEEE
}

\begin{abstract}
A closed-form expression is presented in this brief to accurately estimate the effective inductance of a single layer within an interdigitated power and ground (P/G) distribution network. Due to the large number of $P / G$ lines in these networks, excessive time is required to calculate the inductance using 3-D simulation tools. The proposed expression is favorably compared with previous models and FastHenry, exhibiting accuracy and computational efficiency. The inductance of a single layer within an interdigitated $P / G$ distribution network is bounded for any number of lines. The error of the proposed expression rapidly decreases with an increasing number of pairs within the network. The upper bound for the error of the proposed model is also determined.
\end{abstract}

Index Terms - Interdigitated structure, power and ground (P/G) network, mutual inductance, self-inductance.

\section{INTRODUCTION}

W ITH high operating frequencies and scaled geometries, power and ground $(\mathrm{P} / \mathrm{G})$ distribution networks require greater design optimization to effectively provide higher current flow. Low supply voltages and high currents in ICs place stringent constraints on $\mathrm{P} / \mathrm{G}$ distribution networks. Higher frequencies and smaller transistors produce shorter transition times, such that $L(d i / d t)$ voltage drops exceed $I R$ voltage drops. All of these factors require the inductance to be considered in the design of on-chip P/G distribution networks. To optimize these large-scale $\mathrm{P} / \mathrm{G}$ distribution networks, the inductance needs to be accurately and efficiently calculated.

An interdigitated $\mathrm{P} / \mathrm{G}$ distribution network structure, where a few wide lines are replaced by a large number of narrow lines, is often used to reduce the inductance effect [1], [2]. Different P/G structures have been compared in [3], where the interdigitated structure is shown to achieve the greatest reduction in inductive effects. An interdigitated structure has also been applied to clock networks in [4].

Each layer of a multiplane $\mathrm{P} / \mathrm{G}$ distribution network consists of interdigitated $\mathrm{P} / \mathrm{G}$ lines. The direction of the wires is perpendicular to the direction of the wires in the previous layer, as shown in Fig. 1, ensuring no inductive coupling between the layers. The advantages of an interdigitated structure are in-

Manuscript received January 14, 2009; revised March 27, 2009. First published June 16, 2009; current version published July 17, 2009. This work was supported in part by the National Science Foundation under Grant CCF0541206, Grant CCF-0811317, and Grant CCF-0829915, by grants from the New York State Office of Science, Technology and Academic Research to the Center for Advanced Technology in Electronic Imaging Systems, and by grants from Intel Corporation, Eastman Kodak Company, and Freescale Semiconductor Corporation. This paper was recommended by Associate Editor P. Li.

The authors are with the Department of Electrical Engineering, University of Rochester, Rochester, NY 14627-0231 USA (e-mail: jakushok@ece. rochester.edu; friedman@ece.rochester.edu).

Digital Object Identifier 10.1109/TCSII.2009.2023297

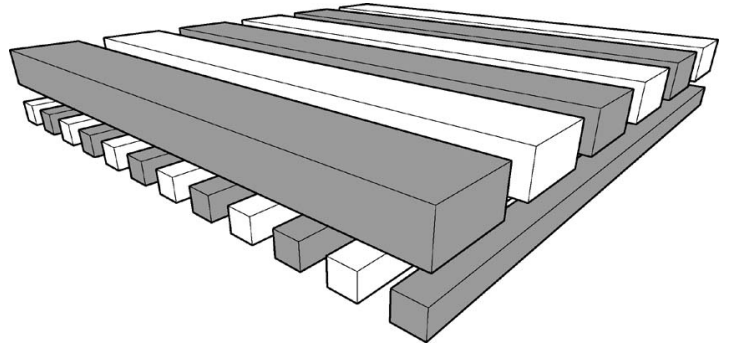

Fig. 1. Interdigitated $\mathrm{P} / \mathrm{G}$ distribution network. The darker and lighter lines represent the power and ground lines, respectively.
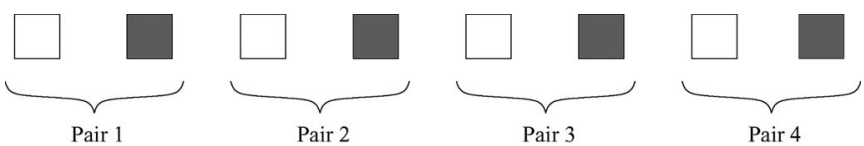

Fig. 2. Four pairs of a single layer within an interdigitated $P / G$ distribution network.

creased routing flexibility and reduced inductance effects. The current flow of the $P / G$ lines within a layer is assumed to flow in opposite directions, thereby reducing the loop inductance of the network [5]. The loop inductance of two parallel wires with opposite current flow is

$$
L_{\text {loop }}=L_{11}+L_{22}-2 M_{12}
$$

where $L_{11}, L_{22}$, and $M_{12}$ are the self-inductance of the P/G lines and the mutual inductance between these two wires, respectively.

The process of estimating the inductance becomes problematic with a large number of wires. To calculate the loop inductance, the mutual inductance terms among all of the wires need to be individually determined, which is a computationally expensive process. A closed-form expression characterizing this inductance would therefore be useful.

The inductance of a single layer within an interdigitated P/G distribution network structure with four pairs (eight wires), as shown in Fig. 2, is

$$
\frac{1}{L_{\mathrm{eff}}}=\frac{1}{L_{1}}+\frac{1}{L_{2}}+\frac{1}{L_{3}}+\frac{1}{L_{4}}
$$

where $L_{1}, L_{2}, L_{3}$, and $L_{4}$ are the inductance of the first, second, third, and fourth pairs of a single layer within a P/G distribution network, respectively. The inductance of the first pair is

$$
\begin{aligned}
L_{1}=L_{1_{p}}+L_{1_{g}}+\sum_{i=2}^{4}\left(M_{1_{p} i_{p}}+\right. & \left.M_{1_{g} i_{g}}\right) \\
& -\sum_{i=1}^{4}\left(M_{1_{p} i_{g}}+M_{1_{g} i_{p}}\right)
\end{aligned}
$$




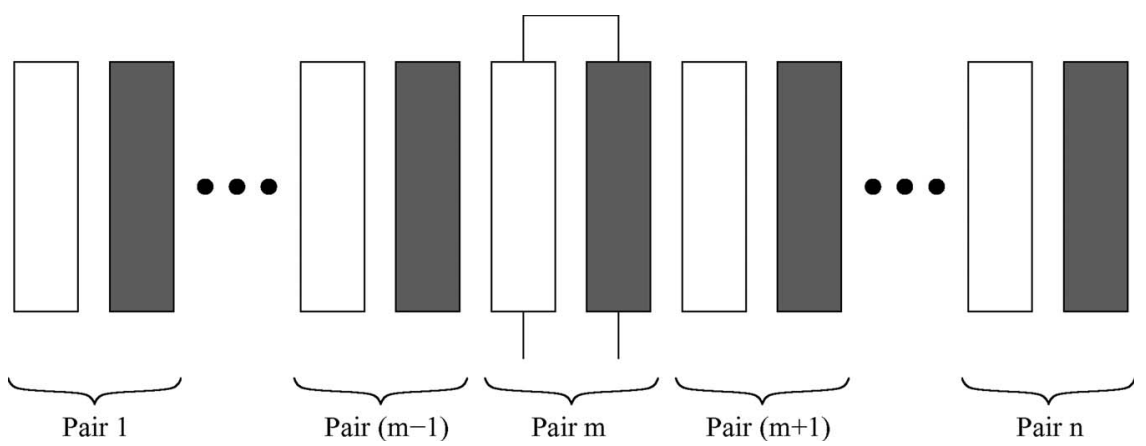

Fig. 3. $n$ pairs of a P/G distribution network. The focus of (10) is on the effective inductance of pair $m$.

where subscripts $p$ and $g$ represent power and ground, respectively. In this case, the overall inductance requires sixteen terms to determine each pair. For $n$ pairs of $\mathrm{P} / \mathrm{G}$ distribution networks, $2 \cdot 2 n=4 n$ terms are required to characterize each pair, making complexity $O(n)$ for a single pair. For $n$ pairs, the complexity in estimating the inductance of a single layer within a $\mathrm{P} / \mathrm{G}$ network is $O\left(n^{2}\right)$.

The brief is organized as follows: A closed-form expression of the self- and mutual inductance of an interdigitated P/G distribution network is presented in Section II. The accuracy of this expression and a comparison to other models are provided in Section III. The upper bound for the error of the proposed model is also provided. The brief is concluded in Section IV.

\section{Inductance OF P/G Distribution Network}

The definition of the inductance between two loops $i$ and $j$ for a uniform current density is presented by the Neumann equation

$$
L_{i j} \equiv \frac{\mu_{0} \mu_{r}}{4 \pi} \oint_{C_{i}} \oint_{C_{j}} \frac{d s_{i} d s_{j}}{\left|R_{i j}\right|}
$$

where $\mu_{0}, \mu_{r}$, and $R_{i j}$ are the vacuum and relative permeability, and the distance between two loops, respectively. From [6], the mutual inductance between a pair of two rectangular conductors is

$$
M=\frac{\mu_{0} l}{2 \pi}\left[\ln \left(\frac{l}{d}+\sqrt{1+\frac{l^{2}}{d^{2}}}\right)-\sqrt{1+\frac{d^{2}}{l^{2}}}+\frac{d}{l}\right]
$$

where $l$ and $d$ are the length of the wire and the pitch of two wires, respectively. If $l \gg d$, an approximate expression based on a Taylor series expansion is [7]

$$
M=\frac{\mu_{0} l}{2 \pi}\left[\ln \left(\frac{2 l}{d}\right)-1+\frac{d}{l}\right] .
$$

The self-inductance is derived in a similar way. For those cases where the length is larger than the width [8]

$$
L_{s}=\frac{\mu_{0} l}{2 \pi}\left[\ln \left(\frac{2 l}{w+t}\right)+\frac{1}{2}+\frac{k(w+t)}{l}\right]
$$

where $w, t$, and $k$ are the wire width, wire thickness, and fitting parameter $(k \approx 0.22)$ for smaller length wires, respectively. In $\mathrm{P} / \mathrm{G}$ distribution networks where $l \gg d$ and $l \gg w+t$, the last term characterizing the edge effect of the self- and mutual inductance can be neglected, simplifying (6) and (7) to

$$
\begin{aligned}
& M=\frac{\mu_{0} l}{2 \pi}\left[\ln \left(\frac{2 l}{d}\right)-1\right] \\
& L_{s}=\frac{\mu_{0} l}{2 \pi}\left[\ln \left(\frac{2 l}{w+t}\right)+\frac{1}{2}\right]
\end{aligned}
$$

respectively. The mutual component of the inductance within an interdigitated $\mathrm{P} / \mathrm{G}$ distribution network decreases with increasing distance between the wires and can be treated as a local effect, according to [5]. In this case, the effective inductance of each pair is the sum of the self-inductances and a single mutual inductance between the two wires in the pair. This approach supports fast estimation of the effective inductance of a $P / G$ distribution network but suffers in accuracy since the mutual inductance terms between all other parallel wires are neglected. Enhanced accuracy in estimating the mutual inductance terms is required.

The effective inductance of an arbitrary pair of P/G lines $m$ within an interdigitated $\mathrm{P} / \mathrm{G}$ distribution network is shown in Fig. 3 and is

$$
\begin{aligned}
L_{m}=2 L_{m_{s}} & -2 M_{m_{p} m_{g}}+\sum_{\substack{i=1 \\
i \neq m}}^{n} \\
& \times\left(M_{m_{p} i_{p}}-M_{m_{p} i_{g}}-M_{m_{g} i_{p}}+M_{m_{g} i_{g}}\right) .
\end{aligned}
$$

The terms $M_{m_{p} i_{p}}=M_{m_{g} i_{g}}$ are equal for any $i$ in (10) since the distance between the power lines of pair $m$ and $i$ and the ground lines of pair $m$ and $i$ is the same. In addition, (10) can be rewritten as a function of distance $d=w+s$, where $s$ is the spacing

$$
\begin{aligned}
L_{m}= & 2 L_{m_{s}}-2 M(d) \\
& +\sum_{\substack{i=1 \\
i \neq m \\
k=|m-i|}}^{n}[2 M(2 d k)-M(2 d k-d)-M(2 d k+d)]
\end{aligned}
$$

where

$$
M(x)=\frac{\mu_{0} l}{2 \pi}\left[\ln \left(\frac{2 l}{x}\right)-1\right] .
$$

Equation (11) consists of three terms: 1) the self-inductance of two wires; 2) the mutual inductance between these two wires; and 3 ) the sum of the mutual inductance between all of the other 


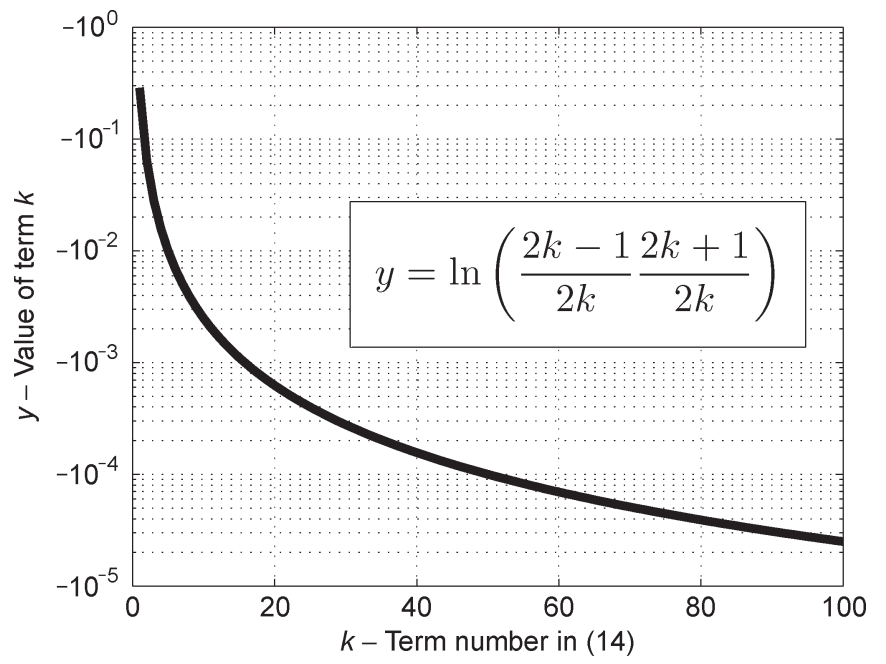

Fig. 4. Terms of (14). The values quickly decline in magnitude to zero.

wires. The third term is neglected in [5]. Substituting (12) into (11), the summation term is

$$
\begin{aligned}
\sum M= & \sum_{\substack{i=1 \\
i \neq m \\
k=|m-i|}}^{n} \frac{\mu_{0} l}{2 \pi} \\
& {\left[2 \ln \left(\frac{2 l}{2 d k}\right)-\ln \left(\frac{2 l}{2 d k-d}\right)-\ln \left(\frac{2 l}{2 d k+d}\right)\right] . }
\end{aligned}
$$

The sum of the logarithmic terms is the product of a single logarithm, permitting (13) to be expressed as

$$
\begin{aligned}
\sum M & =\sum_{\substack{i=1 \\
i \neq m \\
k=|m-i|}}^{n} \frac{\mu_{0} l}{2 \pi} \ln \left(\frac{2 l}{2 d k} \frac{2 l}{2 d k} \frac{2 d k-d}{2 l} \frac{2 d k+d}{2 l}\right) \\
& =\sum_{\substack{i=1 \\
i \neq m \\
k=|m-i|}}^{n} \frac{\mu_{0} l}{2 \pi} \ln \left(\frac{2 d k-d}{2 d k} \frac{2 d k+d}{2 d k}\right) \\
& =\frac{\mu_{0} l}{2 \pi} \sum_{\substack{i=1 \\
i \neq m \\
k=|m-i|}}^{n} \ln \left(\frac{2 k-1}{2 k} \frac{2 k+1}{2 k}\right) .
\end{aligned}
$$

$\mathrm{P} / \mathrm{G}$ distribution networks typically consist of a large number of interdigitated pairs, and, as shown in Fig. 4, the terms of (14) quickly decline in magnitude to zero. The number of pairs on the left (and right) is therefore assumed to be infinite, permitting (14) to be formulated as

$$
\sum M=\frac{\mu_{0} l}{2 \pi} \lim _{n \rightarrow \infty}\left[2 \sum_{k=1}^{n} \ln \left(\frac{2 k-1}{2 k} \frac{2 k+1}{2 k}\right)\right] .
$$

The factor of two originates from the two sides of the target pair. The infinite sum of (15) is presented as an infinite product

$$
\begin{aligned}
\sum M & =2 \frac{\mu_{0} l}{2 \pi} \ln \left[\lim _{n \rightarrow \infty} \prod_{k=1}^{n}\left(\frac{2 k-1}{2 k} \frac{2 k+1}{2 k}\right)\right] \\
& =2 \frac{\mu_{0} l}{2 \pi} \ln \left[\lim _{n \rightarrow \infty} \prod_{k=1}^{n}\left(1-\frac{1}{(2 k)^{2}}\right)\right] .
\end{aligned}
$$

The limit of the product can be solved using the Wallis formula [9]

$$
\frac{\sin (x)}{x}=\prod_{n=1}^{\infty}\left(1-\frac{x^{2}}{\pi^{2} n^{2}}\right)
$$

at $x=\pi / 2$, leading to the equality

$$
\lim _{n \rightarrow \infty} \prod_{k=1}^{n}\left(1-\frac{1}{(2 k)^{2}}\right)=\frac{2}{\pi} \text {. }
$$

Based on (18), (10) may be presented in closed form as

$$
\begin{aligned}
L_{m} & =2 \frac{\mu_{0} l}{2 \pi}\left[\ln \left(\frac{2 l}{w+t}\right)+\frac{1}{2}-\ln \left(\frac{2 l}{d}\right)+1+\ln \left(\frac{2}{\pi}\right)\right] \\
& =2 \frac{\mu_{0} l}{2 \pi}\left[\ln \left(\frac{d}{w+t}\right)+\frac{3}{2}+\ln \left(\frac{2}{\pi}\right)\right] .
\end{aligned}
$$

To estimate the overall inductance of a structure with $N$ $\mathrm{P} / \mathrm{G}$ line pairs, the inductance of each pair is assumed to be equal. The mutual inductance between all of the other $P / G$ pairs converges to a constant, making the inductance independent of the number of $\mathrm{P} / \mathrm{G}$ pairs. The error is greatest in those cases where the number of pairs is smallest; however, in these cases, the effective inductance can quickly be determined with no approximation due to the small number of pairs. For those cases where the number of pairs is sufficiently large (eight pairs produce less than $10 \%$ error), the effective inductance is

$$
L_{\mathrm{eff}}=\frac{2}{N} \frac{\mu_{0} l}{2 \pi}\left[\ln \left(\frac{d}{w+t}\right)+\frac{3}{2}+\ln \left(\frac{2}{\pi}\right)\right] .
$$

Note than the effective inductance is described for a single layer within an interdigitated $\mathrm{P} / \mathrm{G}$ network, where it is assumed that no metal layers are above or below the structure. In practical cases, the existence of different interconnect structures above or below the structure may reduce the accuracy of the proposed model. For structures with large spacing, an interconnect structure below the target structure reduces the accuracy of the estimated inductance [10]. Interdigitated P/G networks, however, are designed with small spacing to exploit the available metal resources; therefore, the accuracy of the effective inductance model is maintained.

Additionally, since the current is assumed to flow throughout the entire interdigitated structure, the inductance determined in (20) represents the worst case effective inductance. Assuming that the current is uniformly distributed throughout the interdigitated structure, the worst case effective inductance produces the largest voltage drop over the $\mathrm{P} / \mathrm{G}$ distribution network.

\section{COMPARISON AND DISCUSSION}

Three different models are compared in this section. The Grover model describes the inductance of each pair based on (10), where every mutual component is individually calculated [7]. While the individual inductance of each pair is determined, the effective inductance of a single layer within an interdigitated $\mathrm{P} / \mathrm{G}$ network structure is estimated, assuming that the individual inductive lines are in parallel. Hence, the Grover model refers to the evaluation of every mutual term among all of the wires 


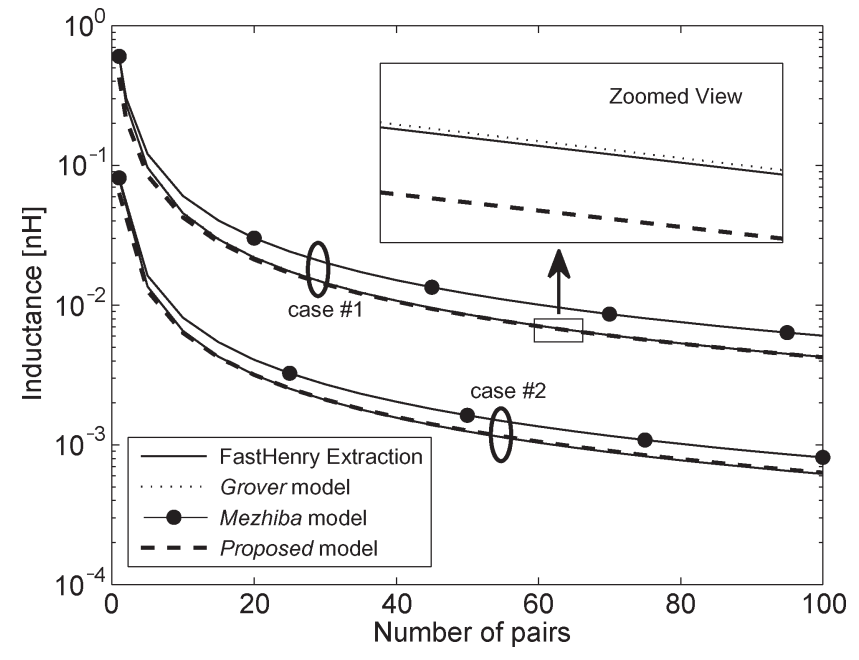

Fig. 5. Comparison of FastHenry and the Grover, Mezhiba, and proposed models for two different design cases.

in a system. In [5], the effective inductance is determined based on an approximation, where the inductance is treated as a local effect, and the mutual inductance between other pairs is neglected. This model is called the Mezhiba model. The proposed model, as represented by (20), determines the effective inductance, assuming that the number of $P / G$ pairs is infinite. Since the magnitude of the mutual terms quickly declines to zero as a function of distance, this assumption is highly accurate.

A comparison among FastHenry [11] (which is a multipole 3-D inductance extraction program) and the Grover, Mezhiba, and proposed models is summarized in this section. In addition, the complexity and accuracy of the proposed, Grover, and Mezhiba models are compared. Two different structures of an interdigitated $\mathrm{P} / \mathrm{G}$ distribution network are evaluated. For both structures, the width and spacing are maintained constant, i.e., $w=1 \mu \mathrm{m}$ and $s=1 \mu \mathrm{m}$; however, the length and thickness are different, i.e., $l_{1}=1 \mathrm{~mm}, t_{1}=0.975 \mu \mathrm{m}$, and $l_{2}=100 \mu \mathrm{m}$, $t_{2}=0.17 \mu \mathrm{m}$. The thickness values are based on a $65-\mathrm{nm}$ CMOS technology [12] for the top (M8) and bottom (M1) metal layers, respectively. Both cases represent a single layer within a P/G distribution network. In Fig. 5, two structures are extracted using FastHenry and compared with the Grover, Mezhiba, and proposed models. The proposed and Grover models exhibit enhanced accuracy as compared with the Mezhiba model. In Figs. 6 and 7, the accuracy and complexity are evaluated, respectively. The accuracy is evaluated by comparing the results with FastHenry. The complexity of FastHenry is, however, not evaluated since the required number of terms for the simulator is excessively large as compared with the analytic model.

The complexity and error of the Grover, Mezhiba, and proposed models relative to FastHenry are evaluated. The Grover model considers all of the mutual terms and exhibits the lowest error (less than $1 \%$ error); however, the complexity of the Grover model drastically increases for a large number of pairs. The complexity of the proposed and Mezhiba models is independent of the number of $\mathrm{P} / \mathrm{G}$ pairs. The error of the proposed model decreases with a larger number of $P / G$ pairs, whereas the error of the Mezhiba model increases. The highest error $(\sim 30 \%)$ of the proposed model occurs with the fewest number of pairs, whereas the error of the Mezhiba model is

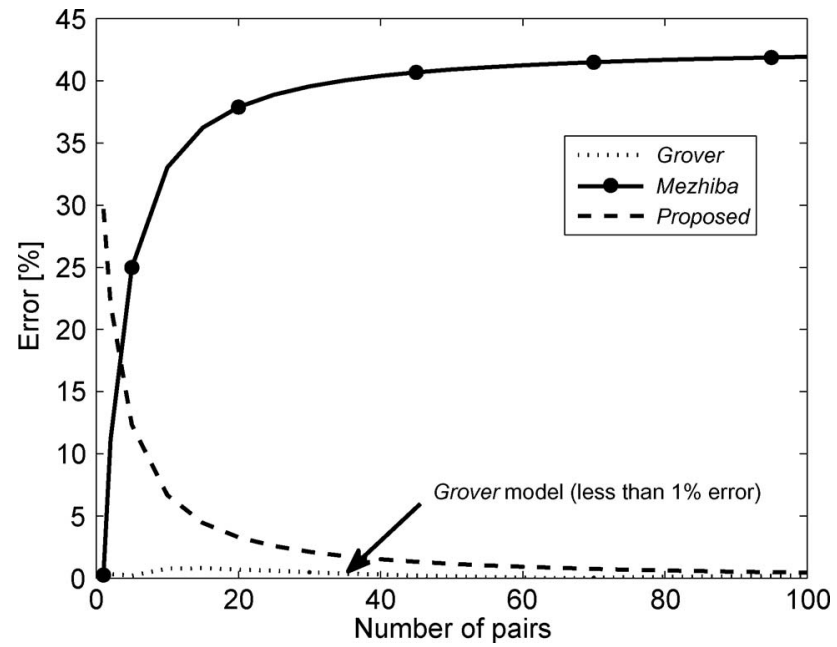

Fig. 6. Error comparison for the Grover, Mezhiba, and proposed models. All of the models are compared with FastHenry.

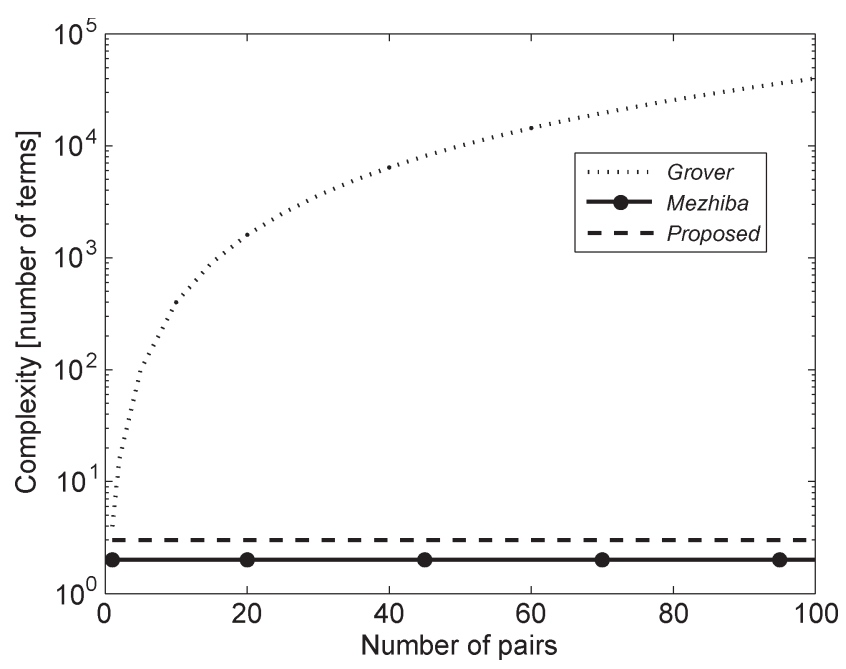

Fig. 7. Comparison of complexity of the Grover, Mezhiba, and proposed models.

highest with the greatest number of $\mathrm{P} / \mathrm{G}$ pairs. Hence, the error of the proposed model can be reduced using the Grover model, which is only computationally efficient for a few P/G pairs.

Assuming that the number of $\mathrm{P} / \mathrm{G}$ pairs is infinite, the effect of the mutual inductance terms is greater; therefore, the proposed model underestimates the effective inductance. The mutual inductance of only a single pair is considered by the Mezhiba model, overestimating the inductance. The boundary conditions of the effective inductance are determined from the proposed and Mezhiba models. These conditions permit the effective inductance of a single layer within an interdigitated $\mathrm{P} / \mathrm{G}$ distribution network structure to be determined for any number of $\mathrm{P} / \mathrm{G}$ line pairs. The boundary conditions for the effective inductance of an interdigitated $\mathrm{P} / \mathrm{G}$ distribution network structure are therefore determined by the proposed and Mezhiba models

$$
\begin{aligned}
\frac{2}{N} \frac{\mu_{0} l}{2 \pi}\left[\ln \left(\frac{d}{w+t}\right)+\frac{3}{2}+\ln \left(\frac{2}{\pi}\right)\right] \\
\leq L_{\mathrm{eff}}(x) \leq \frac{2}{N} \frac{\mu_{0} l}{2 \pi}\left[\ln \left(\frac{d}{w+t}\right)+\frac{3}{2}\right]
\end{aligned}
$$


where $x$ represents any number of pairs within a single layer of an interdigitated $\mathrm{P} / \mathrm{G}$ distribution network.

An expression is derived for the error between the proposed and Grover models. The normalized error is

$$
\text { error }=\left|\frac{L_{\text {grover }}-L_{\text {proposed }}}{L_{\text {grover }}}\right|=\left|1-\frac{L_{\text {proposed }}}{L_{\text {grover }}}\right| \text {. }
$$

Since the Grover model cannot be expressed by a single equation, only the worst case error is determined. An assumption in the proposed model is that the number of interdigitated pairs is infinite; therefore, the error is highest when only a single pair $(N=1)$ is present, expressing error $_{N=n} \leq \operatorname{error}_{N=1}$. For this case, the inductance based on the Grover model is

$$
L_{\text {grover }(N=1)}=2 L_{s}-2 M=\frac{2 \mu_{0} l}{2 \pi}\left[\ln \left(\frac{d}{w+t}\right)+\frac{3}{2}\right] .
$$

The inductance based on the proposed model for $N=1$ is

$$
L_{\text {proposed }(N=1)}=\frac{2 \mu_{0} l}{2 \pi}\left[\ln \left(\frac{d}{w+t}\right)+\frac{3}{2}+\ln \left(\frac{2}{\pi}\right)\right] .
$$

Substituting (23) and (24) into (22), the error bound is

$$
\text { ErrorBound }_{N \geq 1}=\frac{\ln \left(\frac{\pi}{2}\right)}{\ln \left(\frac{d}{w+t}\right)+\frac{3}{2}} .
$$

Based on the parameters of width, spacing, and thickness provided earlier in this brief, the ErrorBound is less than 0.3 or $30 \%$. The error of the proposed model drastically decreases with a higher number of pairs, as shown in Fig. 6. Similarly, the Error Bound can be expressed for those cases where $N \geq 2$

$$
\text { ErrorBound }_{N \geq 2}=\frac{\ln \left(\frac{\sqrt{3}}{2} \frac{\pi}{2}\right)}{\ln \left(\frac{d}{w+t}\right)+\frac{3}{2}+\ln \left(\frac{\sqrt{3}}{2}\right)} .
$$

The ErrorBound is less than 0.23 or $23 \%$ for those cases where $N \geq 2$ with the aforementioned parameters of width, spacing, and thickness.

\section{CONCLUSIONS}

Due to the higher operating frequencies, the role of inductance in the IC design process has become significant. Inductance estimation is a complicated task since every mutual inductance within a system must be considered. An interdigitated $\mathrm{P} / \mathrm{G}$ distribution network reduces the effective inductance as compared with other $\mathrm{P} / \mathrm{G}$ distribution network structures. An accurate yet computationally efficient model of this system is desirable to enhance the P/G network design process. The sym- metric structure of an interdigitated $\mathrm{P} / \mathrm{G}$ distribution network supports the development of a closed-form expression to model the effective inductance.

A closed-form expression characterizing the inductance of a single layer within a $\mathrm{P} / \mathrm{G}$ distribution network has been presented. The solution is compared with previous work and FastHenry, exhibiting good accuracy. The error for the proposed model is highest for a few P/G pairs; however, due to the small number of lines, the Grover model can be used in these cases. With an increasing number of $\mathrm{P} / \mathrm{G}$ network pairs, the error of the proposed model rapidly decreases, permitting the effective inductance of a $P / G$ distribution network to be accurately and efficiently estimated. The magnitude of the effective inductance is bounded by the values determined from the proposed and Mezhiba models. The bound dramatically decreases with an increasing number of pairs. The effective inductance of a single layer within an interdigitated $\mathrm{P} / \mathrm{G}$ distribution network structure can therefore be determined for any number of $P / G$ line pairs.

\section{REFERENCES}

[1] D. A. Priore, "Inductance on silicon for sub-micron CMOS VLSI," in Proc. IEEE Symp. VLSI Circuits, May 1993, pp. 17-18.

[2] L.-R. Zheng and H. Tenhunen, "Effective power and ground distribution scheme for deep submicron high speed VLSI circuits," in Proc. IEEE Int. Symp. Circuit Syst., May 1999, vol. I, pp. 537-540.

[3] M. Popovich, A. V. Mezhiba, and E. G. Friedman, Power Distribution Networks With on-Chip Decoupling Capacitors. New York: SpringerVerlag, 2008.

[4] Y. Massaud, S. Majors, T. Bustami, and J. White, "Layout techniques for minimizing on-chip interconnect self inductance," in Proc. IEEE/ACM Des. Autom. Conf., Jun. 1998, pp. 566-571.

[5] A. V. Mezhiba and E. G. Friedman, "Inductance properties of highperformance power distribution grids," IEEE Trans. Very Large Scale Integr. (VLSI) Syst., vol. 10, no. 6, pp. 762-776, Dec. 2002.

[6] E. B. Rosa, "The self and mutual inductances of linear conductors," Bull. Bureau Standards, vol. 4, no. 2, pp. 301-344, 1908.

[7] F. Grover, Inductance Calculation: Working Formulas and Tables. New York: Dover, 1962.

[8] E. B. Rosa and F. W. Grover, Formulas and Tables for Calculation of Mutual and Self-Inductance. Washington, DC: Gov. Printing Office, 1916.

[9] J. Wallis, Opera Mathematica, Oxonii, Leon: Lichfield Academiæ Typographi, 1656.

[10] B. Kleveland, X. Qi, L. Madden, T. Furusawa, R. W. Dutton, M. A. Horowitz, and S. S. Wong, "High-frequency characterization of on-chip digital interconnects," IEEE J. Solid-State Circuits, vol. 37, no. 6, pp. 716-725, Jun. 2002.

[11] M. Kamon, M. J. Tsuk, and J. K. White, "FASTHENRY: A multipoleaccelerated 3-D inductance extraction program," IEEE Trans. Microw. Theory Tech., vol. 42, no. 9, pp. 1750-1758, Sep. 1994.

[12] P. Bai, C. Auth, S. Balakrishnan, M. Bost, R. Brain, V. Chikarmane, R. Heussner, M. Hussein, J. Hwang, D. Ingerly, R. James, J. Jeong, C. Kenyon, E. Lee, S.-H. Lee, N. Lindert, M. Liu, Z. Ma, T. Marieb, A. Murthy, R. Nagisetty, S. Natarajan, J. Neirynck, A. Ott, C. Parker, J. Sebastian, R. Shaheed, S. Sivakumar, J. Steigerwald, S. Tyagi, C. Weber, B. Woolery, A. Yeoh, K. Zhang, and M. Bohr, "A $65 \mathrm{~nm}$ logic technology featuring $35 \mathrm{~nm}$ gate lengths, enhanced channel strain, $8 \mathrm{Cu}$ interconnect layers, low-k ILD and $0.57 \mu \mathrm{m}^{2}$ SRAM cell," in IEDM Tech. Dig., Dec. 2004, pp. 657-660. 